\title{
How Does Interference Dynamics Influence Packet Delivery in Cooperative Relaying?
}

\author{
Udo Schilcher \\ University of Klagenfurt \\ Networked and Embedded \\ Systems \\ Klagenfurt, Austria \\ udo.schilcher@aau.at
}

\author{
Stavros Toumpis \\ Athens University of \\ Economics and Business \\ Department of Informatics \\ Athens, Greece \\ toumpis@aueb.gr
}

\author{
Alessandro Crismani \\ University of Klagenfurt \\ Networked and Embedded \\ Systems \\ Klagenfurt, Austria \\ alessandro.crismani@aau.at
}

\author{
Günther Brandner \\ University of Klagenfurt \\ Networked and Embedded \\ Systems \\ Klagenfurt, Austria \\ guenther.brandner@aau.at
}

\author{
Christian Bettstetter \\ University of Klagenfurt \\ Networked and Embedded \\ Systems \\ Lakeside Labs $\mathrm{GmbH}$ \\ Klagenfurt, Austria
}

\begin{abstract}
We show by means of stochastic geometry that interference dynamics has a strong impact on the performance of cooperative relaying. For both conventional and multi-hopaware cooperative relaying we show that the packet delivery probability significantly changes with the dependence of interference among the links. Depending on the scenario under consideration, this change could be either an increase or decrease of the packet delivery probability. Especially for multi-hop-aware cooperative relaying, the performance gain is heavily reduced when interference possesses high temporal and spatial dependence.
\end{abstract}

\section{Categories and Subject Descriptors}

C.2.1 [Computer-communication networks]: Network architecture and design-wireless communication, network communications; G.3 [Probability and statistics]: Stochastic processes

\section{General Terms}

Performance, Theory

\section{Keywords}

Cooperative relaying; interference; diversity; multi-hopaware relaying; fading.
Permission to make digital or hard copies of part or all of this work for personal or classroom use is granted without fee provided that copies are not made or distributed for profit or commercial advantage, and that copies bear this notice and the full citation on the first page. Copyrights for third-party components of this work must be honored. For all other uses, contact the owner/author(s). Copyright is held by the author/owner(s).

MSWiM'13, November 3-8, 2013, Barcelona, Spain

ACM 978-1-4503-2353-6/13/11

http://dx.doi.org/10.1145/2507924.2507926.

\section{INTRODUCTION AND MOTIVATION}

The behavior of interference in wireless networks has a huge impact on system design and performance $[9,14]$. In simple terms, interference is caused if, for a given transmitter-receiver pair, at least one additional node is transmitting at the same time and at the same carrier frequency in the vicinity of the receiver. The signals of all such nodes interfere at the receiver with the intended signal from the transmitter due to the broadcast nature of the wireless medium. Such co-channel interference limits the overall network capacity in terms of bit $/ \mathrm{s} / \mathrm{Hz} / \mathrm{m}^{2}[10]$. Besides the average interference level, the time-varying and space-varying properties of interference should be considered for the design of communication techniques and protocols, and notably for temporal and spatial diversity schemes [21]. If, e.g., a basic scheme retransmits lost packets, this might lead to problems for highly dependent interference. In such scenarios interference is unlikely to change significantly between two transmissions and hence the retransmission is likely to fail.

Along this line of argumentation, this paper applies methods from spatial stochastics to analyze the impact of interference dynamics on cooperative diversity [15] (also called cooperative relaying) with simple selection combining. Most of the research in the past analyzed cooperative relaying without considering such interference dynamics.

In particular, we present the following contributions:

- We extend previous results on the time-varying behavior of packet delivery under interference [3] to the spatio-temporal case.

- We derive the packet delivery probability of a cooperative relaying scenario with selection combining. Additionally, we compare the performance of such relaying under dependent and independent interference for several channel conditions.

- Finally, we analyze multi-hop-aware cooperative relaying [1] assuming that interference is spatially dependent, and compare our results with the case where interference is assumed to be independent. 
The organization of the paper is as follows: Section 2 presents the models and assumptions used for the analysis. Based on these, in Section 3, we derive the spatiotemporal dependence of packet delivery at different locations and time slots by obtaining the joint probability that a number of transmissions is successful under dependent interference. Next, in Section 4, we use these results to calculate the packet delivery probability in a cooperative relaying scenario where the destination relies on selection combining. This analysis is done for both the single iteration and the multiple iterations cases. In Section 5 we calculate the packet delivery probability for multi-hop-aware cooperative relaying [1]. Here, the main focus of our analysis lies in comparing the protocol's performance gain for the dependent and independent interference scenarios. Finally, Section 6 presents an overview of the related work and Section 7 concludes.

\section{MODELING ASSUMPTIONS}

\subsection{Node Setup and Channel Characteristics}

Consider a wireless scenario where nodes are distributed according to a Poisson point process (PPP) $\Phi$ with density $\lambda[19]$. For simplicity, let $\Phi$ denote both the PPP and the nodes' locations. Time is divided into time slots, and each node transmits in each slot with probability $p$. This is similar to random access with slotted ALOHA.

For a given slot $t$, we denote the transmitter by $s(t)$ and the receiver by $d(t)$. In general, also other nodes besides $s(t)$ are transmitting at slot $t$, which causes interference at $d(t)$. The set of all nodes transmitting at slot $t$ is denoted by $\Psi(t) \subseteq \Phi$; the density of the thinned process $\Psi$ is $\lambda p$. For simplicity of notation we often skip the index $t$. The symbols $s$ and $d$ indicate both the nodes and their coordinates.

The channel is modeled using a distance-dependent path loss, where transmissions are subject to Rayleigh fading. Hence, when the node $s$ sends a packet to the node $d$, the signal power received at $d$ is

$$
S_{d}=h(s, d) g(\|s-d\|) .
$$

The function $g(\|s-d\|)$ represents the path loss between the transmitter $s$ and the receiver $d$. We apply the path loss function $g(\|s-d\|)=\|s-d\|^{-\alpha}$, where $\alpha$ is the path loss exponent. The random values $h(s, d)$ model Rayleigh fading caused by multipath propagation. These values are independent and exponentially distributed and have a mean of unity. We have

$$
\mathrm{P}[h \geq \alpha]=\exp (-\alpha) .
$$

The interference level at $d$ in a given slot is the sum of all power values from all interfering signals arriving at $d$, i.e.,

$$
I_{d}=\sum_{u \in \Psi \backslash\{s\}} h(u, d) g(\|u-d\|)
$$

with $u$ indicating both a particular interferer and its position.

The signal-to-interference ratio at the receiver $d$ is

$$
\operatorname{SIR}_{d}=\frac{S_{d}}{I_{d}}=\frac{h(s, d) g(\|s-d\|)}{\sum_{u \in \Psi \backslash\{s\}} h(u, d) g(\|u-d\|)} .
$$

We assume interference to be much higher than noise and therefore omit the noise term.

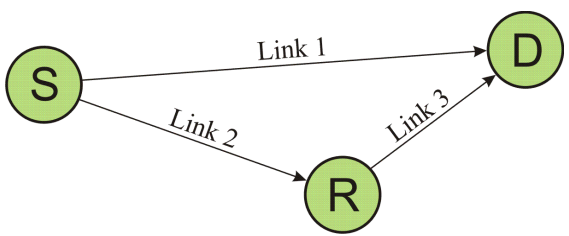

Figure 1: Relaying scenario.

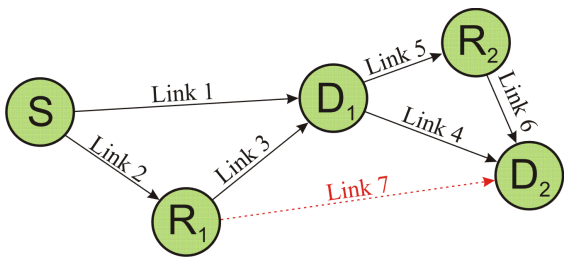

Figure 2: Multi-hop-aware relaying scenario.

Let $A$ denote the event that a packet transmission over the link from a node $s$ to a node $d$ at time slot $t$ is successful. We assume that the transmitter technology used is such that the event $A$ occurs if and only if $\operatorname{SIR}_{d} \geq \theta$, where $\theta$ denotes the receiver threshold. During the operation of the network, in general, several such success events occur for different transmitters and different receivers. A set of $N$ such events is denoted by $\left\{A_{1}, A_{2}, \ldots, A_{i}, \ldots, A_{N}\right\}$. To avoid misinterpretation, note that $i$ is an identifier of the event, neither a time index nor an identifier of the receiver.

\subsection{Cooperative Relaying Schemes}

In this paper we study both conventional cooperative relaying with a single relay [18] and multi-hop-aware (MHA) cooperative relaying [1]. In the conventional relaying system (Figure 1), the relay is assumed to be selected in advance and used over several slots. The receiver decodes the signals received from the transmitter and the relay using selection combining. It works as follows: Node $S$ transmits a data packet to $D$. The relay $R$ overhears this transmission and tries to decode the packet. If the decoding is successful, the relay forwards the packet to $D$. The whole process is successful if $D$ is able to decode at least one of the two transmissions. Note that in our model the relay always forwards the packet independent of whether the direct link succeeds or not. The relaying procedure is described in Algorithm 1.

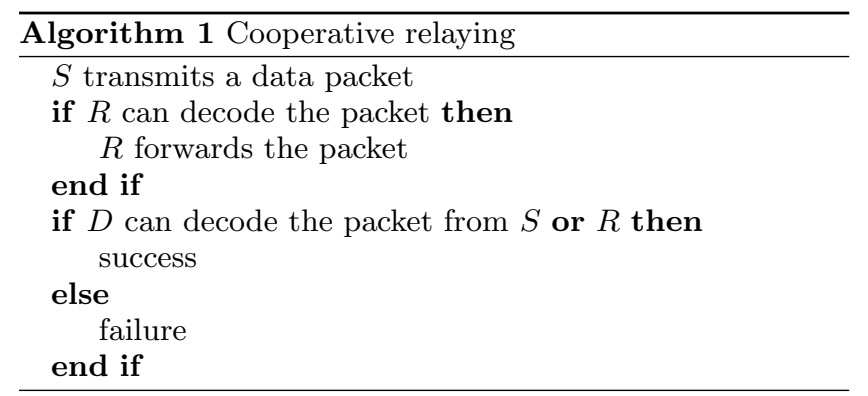

In MHA relaying each relay along a multi-hop path is selected by considering its ability to serve the following two hops. In this paper we show results for a two-hop example, however the analysis is general and may be applied to scenarios consisting of many hops. The two-hop example de- 
picted in Figure 2 works as follows. First, $S$ sends its packet to $D_{1}$. The relay $R_{1}$ overhears the packet and retransmits it. This retransmission might be overheard by the receiver of the second hop (node $D_{2}$ ). Next, $D_{1}$ sends the packet to $D_{2}$. The relay $R_{2}$ tries to decode this transmission and, in the case of success, retransmits the packet. Finally, the scheme is successful if $D_{2}$ receives at least one of the packets from $R_{1}, D_{1}$, and $R_{2}$ correctly. Here, Link 7 in Figure 2 is the link that distinguishes MHA relaying from the conventional two-hop cooperative relaying. The MHA relaying procedure is detailed in Algorithm 2. The motivation for such a setup is that the path over $S, D_{1}$ to $D_{2}$ can be kept stable for a long time. It has to be altered only if a node fails. However, the relays can be reselected each time the channel changes significantly.

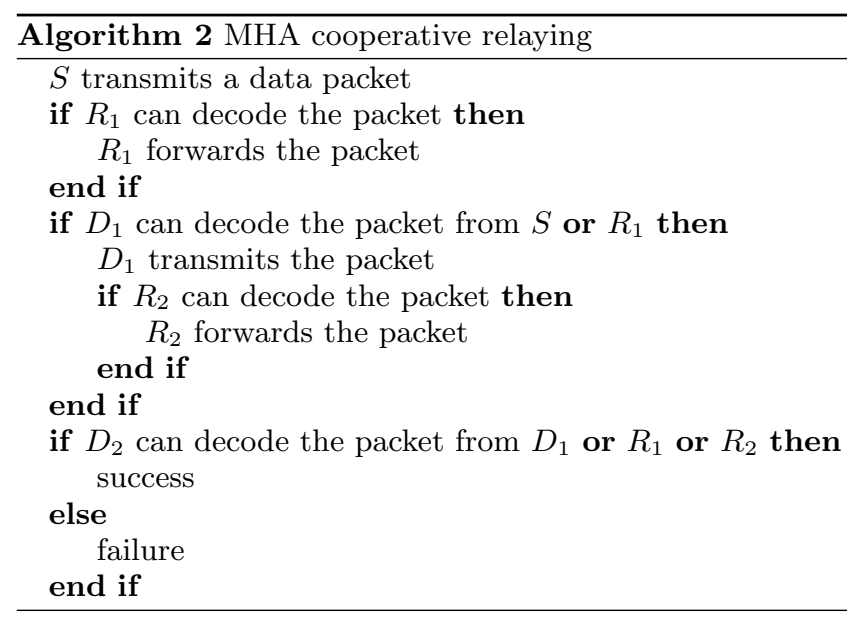

\section{SPATIO-TEMPORAL DEPENDENCE OF TRANSMISSION SUCCESS}

First, we calculate the joint probability $\mathrm{P}\left[A_{1} A_{2} \cdots A_{N}\right]$ that $N$ arbitrary transmissions in the network are successful. To do so, we follow the approach of [3], where Ganti and Haenggi calculate the joint probability for packet delivery over a given link at two or more different time slots. We extend this result by deriving the joint probability for packet delivery over both different time slots and different links.

Observe that

$$
\begin{aligned}
& \mathrm{P}\left[A_{1} \ldots A_{N}\right]= \\
& \quad \mathrm{P}\left[\frac{h\left(s_{1}, d_{1}\right) g\left(\left\|s_{1}-d_{1}\right\|\right)}{\sum_{u \in \Phi} h\left(u, d_{1}\right) g\left(\left\|u-d_{1}\right\|\right) \mathbf{1}\left(u \in \Psi \backslash\left\{s_{1}\right\}\right)} \geq \theta, \ldots,\right. \\
& \left.\quad \frac{h\left(s_{N}, d_{N}\right) g\left(\left\|s_{N}-d_{N}\right\|\right)}{\sum_{u \in \Phi} h\left(u, d_{N}\right) g\left(\left\|u-d_{N}\right\|\right) \mathbf{1}\left(u \in \Psi \backslash\left\{s_{N}\right\}\right)} \geq \theta\right], \quad(5)
\end{aligned}
$$

where $\mathbf{1}$ is the indicator function.

By substituting $\theta_{i}:=\frac{\theta}{g\left(\left\|s_{i}-d_{i}\right\|\right)}$ into (5), the $i$ th term in the brackets yields

$$
h\left(s_{i}, d_{i}\right) \geq \theta_{i} \sum_{u \in \Phi} h\left(u, d_{i}\right) g\left(\left\|u-d_{i}\right\|\right) \mathbf{1}\left(u \in \Psi \backslash\left\{s_{i}\right\}\right) .
$$

From the independence of the fading terms $h$ and (2) we get

$$
\mathrm{P}\left[h\left(s_{1}, d_{1}\right) \geq \alpha_{1}, \ldots, h\left(s_{N}, d_{N}\right) \geq \alpha_{N}\right]=\prod_{i=1}^{N} \exp \left(-\alpha_{i}\right) .
$$

Applying this expression in (5) yields

$$
\begin{aligned}
& \mathrm{P}\left[A_{1} \ldots A_{N}\right]= \\
& \stackrel{(a)}{=} \mathrm{E}_{\Phi, h}\left[\prod _ { i = 1 } ^ { N } \operatorname { e x p } \left(-\theta_{i} \sum_{u \in \Phi} h\left(u, d_{i}\right) g\left(\left\|u-d_{i}\right\|\right)\right.\right. \\
& \left.\left.\mathbf{1}\left(u \in \Psi \backslash\left\{s_{i}\right\}\right)\right)\right] \\
& =\mathrm{E}_{\Phi, h}\left[\prod _ { u \in \Phi } \prod _ { i = 1 } ^ { N } \operatorname { e x p } \left(-\theta_{i} h\left(u, d_{i}\right) g\left(\left\|u-d_{i}\right\|\right)\right.\right. \\
& \left.\left.\quad \mathbf{1}\left(u \in \Psi \backslash\left\{s_{i}\right\}\right)\right)\right] \\
& \quad \mathrm{E}_{\Phi}\left[\prod _ { u \in \Phi } \prod _ { i = 1 } ^ { N } \mathrm { E } _ { h } \left[\operatorname { e x p } \left(-\theta_{i} h\left(u, d_{i}\right) g\left(\left\|u-d_{i}\right\|\right)\right.\right.\right. \\
& \left.\left.\left.\quad \mathbf{1}\left(u \in \Psi \backslash\left\{s_{i}\right\}\right)\right) \mid \Phi\right]\right] \\
& \stackrel{(b)}{=} \mathrm{E}_{\Phi}\left[\prod_{u \in \Phi} \prod_{i=1}^{N}\left(\frac{p}{1+\theta_{i} g\left(\left\|u-d_{i}\right\|\right)}+1-p\right)\right] \\
& \stackrel{(c)}{=} \exp \left(-\lambda \int_{\mathbb{R}^{2}}^{N} 1-\right. \\
& \left.\prod_{i=1}^{N}\left(\frac{p}{1+\theta_{i} g\left(\left\|x-d_{i}\right\|\right)}+1-p\right) \mathrm{d} x\right) .
\end{aligned}
$$

In the above derivation $(a)$ holds since the coefficients $h\left(s_{i}, d_{i}\right)$ are exponentially distributed and independent and we take the expected value over the PPP and the channel coefficients $h\left(u, d_{i}\right)$ of the interferers; in $(b)$ we calculate the expectation over $h\left(u, d_{i}\right) ;(c)$ is due to the probability generating functional of $\Phi$ (cf. [8], (4.8)).

\section{COOPERATIVE RELAYING}

In the following we compute the packet delivery probability of cooperative relaying with selection combining. By considering the dependence of interference, we provide a deeper characterization than related work does (see Sections 6.2 and 6.3). The packet delivery probability is obtained by applying the tools developed in the previous section.

\subsection{Packet Delivery Probability for a Single Iteration}

We consider a three node relaying scenario as depicted in Figure 1. Let $A_{i}$ denote the event that a packet is successfully transmitted via link $i$, with $i \in\{1,2,3\}$. Let $B$ denote the event that the overall scheme is successful, i.e., that $D$ is able to receive at least one of the packets from $S$ or $R$. The overall packet delivery probability can in general be calculated as

$$
\begin{aligned}
\mathrm{P}[B] & =\mathrm{P}\left[A_{1} \cup A_{2} A_{3}\right] \\
& =\mathrm{P}\left[A_{1}\right]+\mathrm{P}\left[A_{2} A_{3}\right]-\mathrm{P}\left[A_{1} A_{2} A_{3}\right]
\end{aligned}
$$

All terms in this equation can be calculated using (8). For the special case of independent interference, we have

$$
\mathrm{P}[B]=\mathrm{P}\left[A_{1}\right]+\mathrm{P}\left[A_{2}\right] \mathrm{P}\left[A_{3}\right]-\mathrm{P}\left[A_{1}\right] \mathrm{P}\left[A_{2}\right] \mathrm{P}\left[A_{3}\right] .
$$




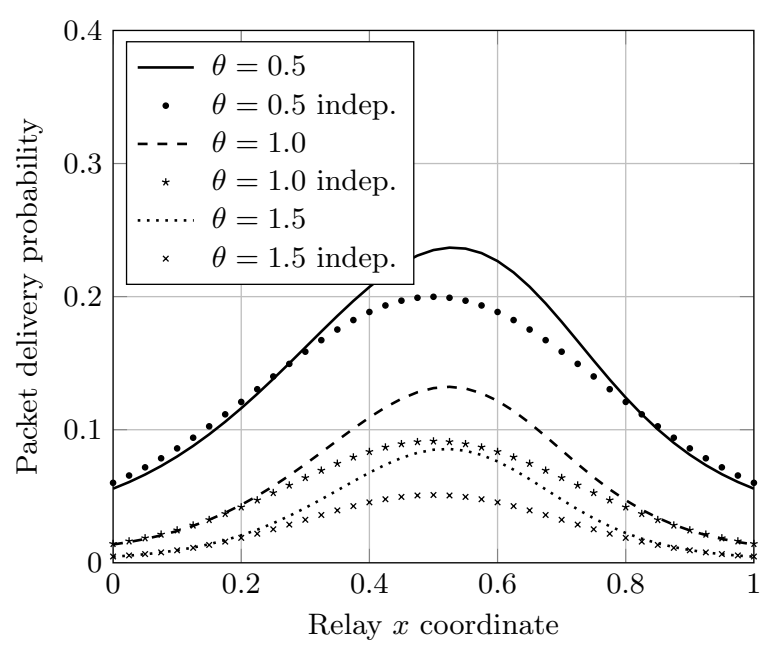

Figure 3: Packet delivery probabilities for cooperative relaying with dependent vs. independent interference over different positions of the relay for an overall transmitter-receiver distance of 1 . Parameters are $\lambda=1, p=1$, and $\alpha=4$.

Figure 3 shows a plot of the packet delivery probability $\mathrm{P}[B]$ for independent interference (marks) given in (10) and for dependent interference (lines) given in (9) when varying the relay's position. We consider a scenario where the transmitter is located at $(0,0)$, the receiver at $(1,0)$ and the relay is placed somewhere on the line between the transmitter and the receiver. Hence, the $y$ coordinate of the relay is 0 , while the $x$ coordinate varies between 0 and 1 .

This scenario leads to very harsh conditions and hence to very low packet delivery probabilities. It has been chosen on purpose to be able to make the following observations.

First, the optimal relay position for independent interference is exactly in the middle between $S$ and $D$. For dependent interference, however, it is slightly closer to $D$.

Second, if $R$ is roughly in the middle between $S$ and $D$, having dependent interference increases the performance. This is because the delivery probability for the two-hop path from $S$ via $R$ to $D$ increases for increasing dependency of interference. This can be best seen in two extreme cases. If interference is independent, we have $\mathrm{P}\left[A_{2} A_{3}\right]=\mathrm{P}\left[A_{2}\right] \mathrm{P}\left[A_{3}\right]$ since the two events are independent. In the opposite case, if interference is highly dependent, we have $\mathrm{P}\left[A_{2} A_{3}\right]=$ $\mathrm{P}\left[A_{2}\right] \mathrm{P}\left[A_{3} \mid A_{2}\right]$. Here, $\mathrm{P}\left[A_{2} A_{3}\right]$ is higher since in the highly dependent case the probability $\mathrm{P}\left[A_{3} \mid A_{2}\right] \gg \mathrm{P}\left[A_{3}\right]$ (see [3]).

Third, if the relay is located close to either $S$ or $D$, the direct $S-D$ link plays a dominant role on the overall performance. In this case, a dependency in interference will decrease performance - if the direct link fails also the twohop path does so and vice versa.

Figure 4 shows results of the same network setup as Figure 3, except that the distance between $S$ and $D$ is halved to 0.5 and $\lambda$ is changed to 0.4 . This significantly improves the performance of the direct link with respect to the twohop path. Therefore, having dependent interference always decreases the performance of the relaying scheme. Additionally, the position of the relay has much less influence on the overall performance compared to the case having an $R-D$ distance equal to unity.

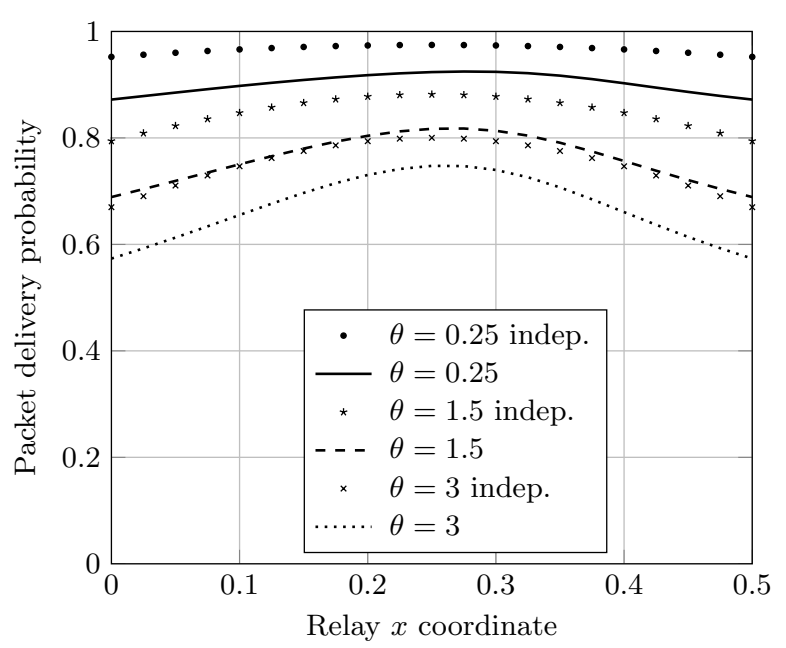

Figure 4: Packet delivery probabilities for cooperative relaying with dependent vs. independent interference for an overall transmitter-receiver distance of 0.5. Parameters are $\lambda=0.4, p=1$, and $\alpha=4$.

Figure 5 shows the packet delivery probability over the transmission probability $p$. In scenarios with weak interference (low $\lambda p$ ), it is better to have independent interference. In scenarios with strong interference (high $\lambda p$ ), dependence helps due to the highly dependent hops of the two-hop path. The explanation is as follows: the direct link between $S$ and $D$ is unlikely to be useful for strong interference while the two-hop path still works due to the multi-hop gain. Thus the increased dependence between direct and two-hop paths plays a less important role than for weak interference. This effect can also be seen in Figure 6, which shows a plot of the

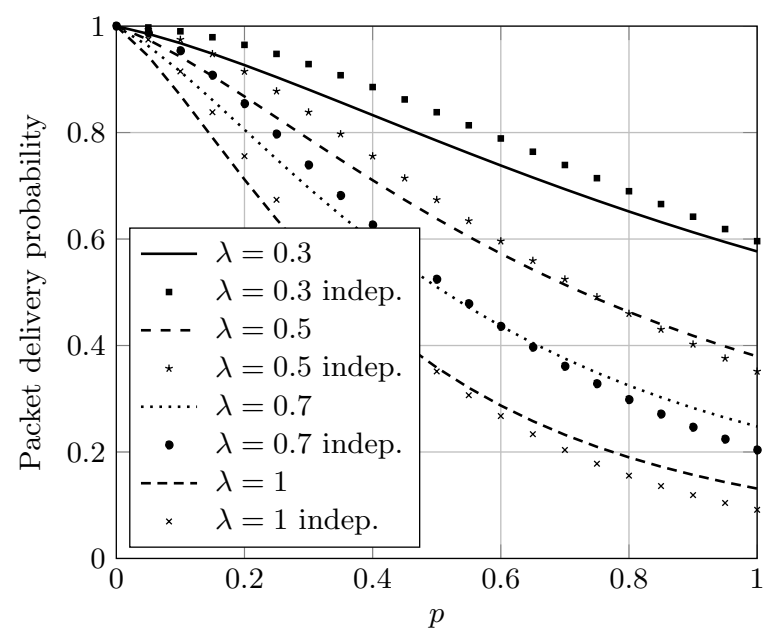

Figure 5: Dependence of packet delivery probabilities for cooperative relaying with dependent vs. independent interference for different sending probabilities $p$. Parameters are $\theta=1, \alpha=4$ and an overall transmitter-receiver distance of 1 , where the relay node is located in the middle between transmitter and receiver. 


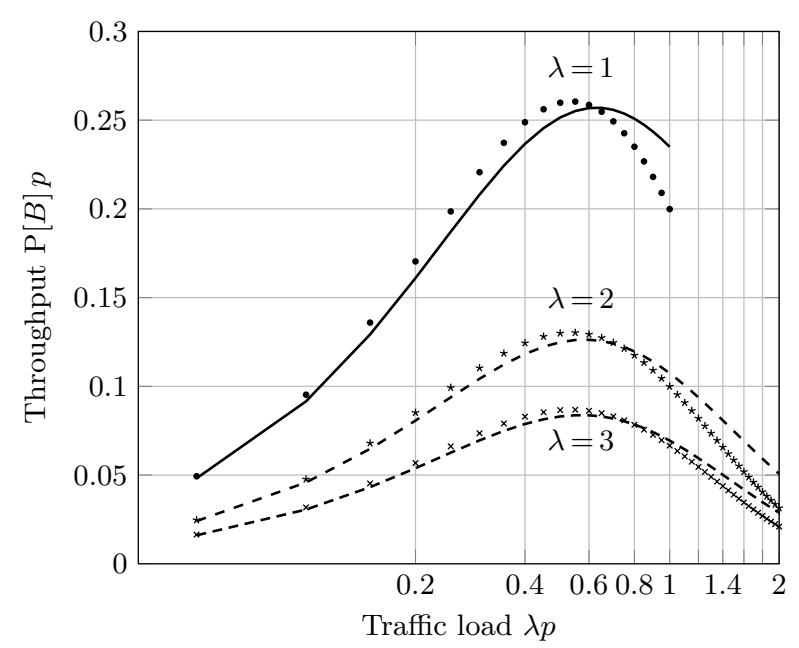

Figure 6: Normalized throughput (in 1 per slot) for cooperative relaying with dependent (lines) vs. independent (marks) interference for different normalized traffic loads $\lambda p$ (in 1 per slot and unit area). Parameters are $\theta=0.5$ and an overall transmitterreceiver distance of 1 , where the relay is located in the middle between transmitter and receiver.

normalized throughput. For higher traffic load the difference between dependent and independent interference cases in terms of throughput increases. Additionally it can be seen that for dependent interference the maximum throughput occurs at a slightly higher traffic load than for independent interference.

\subsection{Packet Delivery Probability for Multiple Iterations}

We now consider a scenario where cooperative relaying is repeated until $D$ receives the packet. In every time slot with an odd number $(2 \nmid t)$ the node $S$ transmits; in every time slot having an even number $(2 \mid t)$, the relay node $R$ transmits assuming it received the transmission from $S$ in the previous slot. Otherwise, the slot stays empty. This procedure is continued until $D$ successfully receives the packet.

In the following, we compute the probability $\mathrm{P}\left[B^{T}\right]$ that the receiver correctly receives the packet at time slot $T$. The event $B^{T}$ occurs if and only if all transmissions in slots $t=1, \ldots, T-1$ are unsuccessful and the transmission in slot $T$ is successful. Let $C(t)$ denote the event that the transmission in slot $t$ is successfully received by $D$, i.e.

$$
C(t)= \begin{cases}A_{1}(t), & \text { if } 2 \nmid t, \\ A_{2}(t-1) \cap A_{3}(t), & \text { if } 2 \mid t .\end{cases}
$$

Here, $A_{i}(t)$ denotes the event that a packet is sent successfully using link $i$ in the relaying scenario (Figure 1) in slot $t$.

$$
\begin{aligned}
\mathrm{P}\left[B^{T}\right] & =\mathrm{P}\left[C(T) \cap \bigcap_{t=1}^{T-1} \bar{C}(t)\right] \\
& =\sum_{\mathcal{T} \subseteq\{1, \ldots, T\}}(-1)^{|\mathcal{T}|} \mathrm{P}\left[C(T) \cap \bigcap_{t \in \mathcal{T}} C(t)\right],
\end{aligned}
$$

where $\bar{C}(t)$ denotes the complementary event of $C(t)$. The

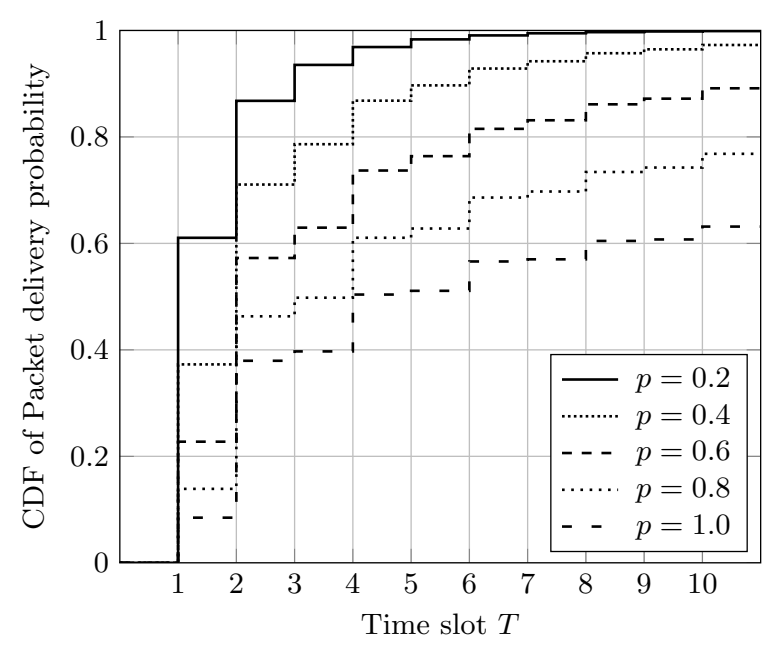

Figure 7: CDF of packet delivery probability $\mathrm{P}\left[B^{T}\right]$ in slot $T$ for different transmit probabilities $p$. Parameters are $\lambda=2, \theta=1, \alpha=4$, an overall transmitter-receiver distance (from $R$ to $D$ ) of $\mathbf{0 . 5}$ and the relay placed in the middle between $R$ and $D$.

last equality holds due to the inclusion-exclusion principle. The joint probabilities can be computed using (8). For independent interference we can simplify this equation to

$$
\mathrm{P}\left[B^{T}\right]=\mathrm{P}[C(T)] \sum_{\mathcal{T} \subseteq\{1, \ldots, T\}}(-1)^{|\mathcal{T}|} \prod_{t \in \mathcal{T}} \mathrm{P}[C(t)]
$$

In Figure 7 the cumulative distribution function (CDF) of the probability $\mathrm{P}\left[B^{T}\right]$ is plotted over $T$ for different interference levels (i.e., for different sending probabilities $p$ ). As can be seen in the plot, the relay transmission significantly increases the packet delivery probability (i.e., the CDF also

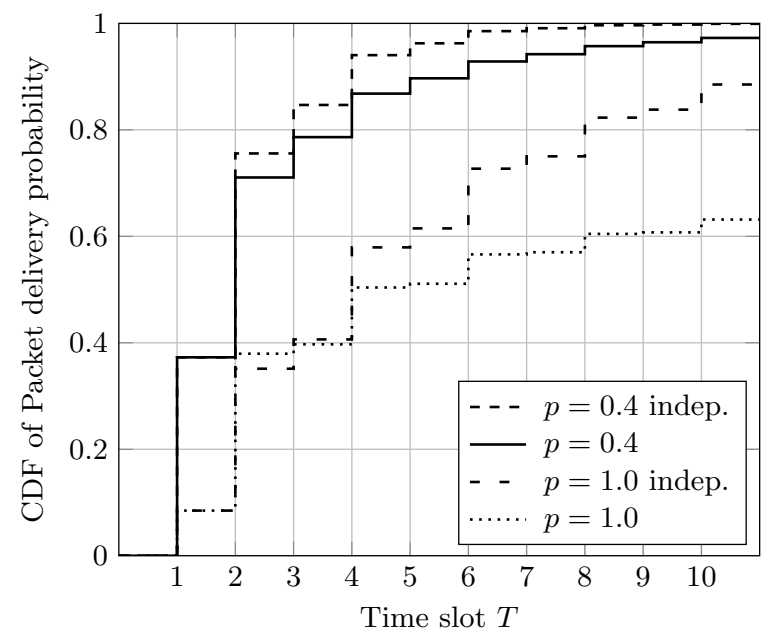

Figure 8: Comparison of the CDFs of the packet delivery probability $\mathrm{P}\left[B^{T}\right]$ in slot $T$ for dependent and independent interference. Parameters are $\lambda=2$, $\theta=1, \alpha=4$, an overall transmitter-receiver distance of 0.5 and the relay is placed in the middle between transmitter and receiver. 


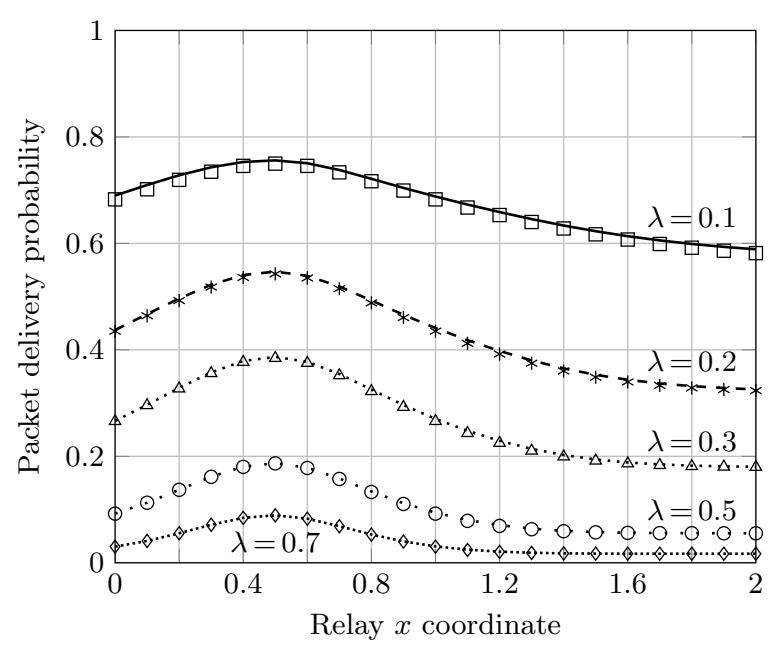

Figure 9: Packet delivery probability of MHA relaying (lines) and dependent two-hop relaying (marks) for different positions of the first relay $R_{1}$. The distance for each $R-D$ hop is 1 and $R_{2}$ is located at 1.5. Parameters are $\theta=1, p=1$, and $\alpha=4$.

increases in even slots). Furthermore, while the first few iterations of the scheme increase the overall packet delivery probability significantly, in later iterations this increase becomes smaller. This smaller increase can still contribute to the overall packet delivery probability, although the retransmission process has to stop at some point. The reduced increase is explained by noting that if the first iteration is unsuccessful, the probability of having success later is small due to dependent interference. If two or more iterations already failed, this effect is even stronger.

Figure 8 compares the packet delivery probabilities $\mathrm{P}\left[B^{T}\right]$ for dependent and independent interference. It can be seen that the CDF converges much faster toward 1 for independent interference than for dependent interference. This shows that the dependence of interference significantly reduces the benefits of retransmissions. This is an example which shows that considering interference dynamics may significantly change the performance of a protocol and therefore must be considered in order for results to be realistic.

\section{MULTI-HOP-AWARE RELAYING}

In the following we study the packet delivery probability of multi-hop-aware cooperative relaying with selection combining [1]. The topology appears in Figure 2.

First, we derive the packet delivery probability $\mathrm{P}[B]$ of a conventional two-hop relaying link, i.e., without using Link 7, for dependent interference. Note that the interference power values of the two hops are dependent, hence we cannot simply square the result in (9). Therefore, we have

$$
\begin{aligned}
\mathrm{P}[B]= & \mathrm{P}\left[\left(A_{1} \cup A_{2} A_{3}\right)\left(A_{4} \cup A_{5} A_{6}\right)\right] \\
= & \mathrm{P}\left[A_{1} A_{4}\right]+\mathrm{P}\left[A_{1} A_{5} A_{6}\right]-\mathrm{P}\left[A_{1} A_{4} A_{5} A_{6}\right] \\
& +\mathrm{P}\left[A_{2} A_{3} A_{4}\right]+\mathrm{P}\left[A_{2} A_{3} A_{5} A_{6}\right] \\
& -\mathrm{P}\left[A_{2} A_{3} A_{4} A_{5} A_{6}\right]-\mathrm{P}\left[A_{1} A_{2} A_{3} A_{4}\right] \\
& -\mathrm{P}\left[A_{1} A_{2} A_{3} A_{5} A_{6}\right]+\mathrm{P}\left[A_{1} A_{2} A_{3} A_{4} A_{5} A_{6}\right] .
\end{aligned}
$$

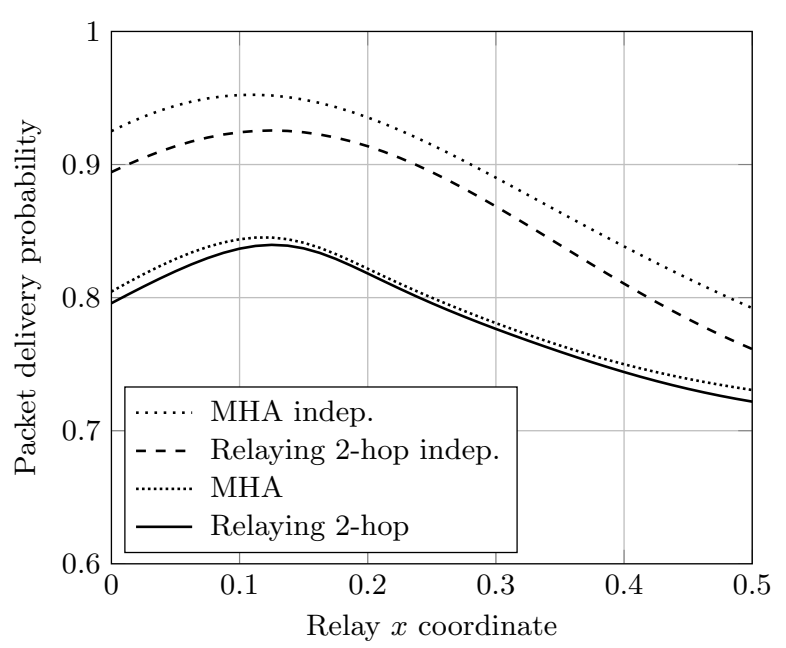

Figure 10: Packet delivery probability of MHA relaying with/without interference dependencies and two-hop relaying with/without dependencies. $\mathbf{P a}-$ rameters are $\lambda=1, \theta=1, p=1, \alpha=4$, and the distance of each $R-D$ hop is 0.25 .

In case of independent interference this simplifies to

$$
\begin{aligned}
\mathrm{P}[B]= & \mathrm{P}\left[\left(A_{1} \cup A_{2} A_{3}\right)\left(A_{4} \cup A_{5} A_{6}\right)\right] \\
= & \left(\mathrm{P}\left[A_{1}\right]+\mathrm{P}\left[A_{2}\right] \mathrm{P}\left[A_{3}\right]-\mathrm{P}\left[A_{1}\right] \mathrm{P}\left[A_{2}\right] \mathrm{P}\left[A_{3}\right]\right) \\
& \left(\mathrm{P}\left[A_{4}\right]+\mathrm{P}\left[A_{5}\right] \mathrm{P}\left[A_{6}\right]-\mathrm{P}\left[A_{4}\right] \mathrm{P}\left[A_{5}\right] \mathrm{P}\left[A_{6}\right]\right)
\end{aligned}
$$

Second, we address the case where Link 7 is also used by node $D_{2}$, i.e., applying MHA cooperative relaying. The overall packet delivery probability for this scenario under dependent interference is given by

$$
\begin{aligned}
& \mathrm{P}\left[B_{\mathrm{MHA}}\right]=\mathrm{P}[B]+\mathrm{P}\left[A_{2} A_{7}\right]-\mathrm{P}\left[A_{1} A_{2} A_{4} A_{7}\right] \\
& \quad-\mathrm{P}\left[A_{2} A_{3} A_{4} A_{7}\right]+\mathrm{P}\left[A_{1} A_{2} A_{3} A_{4} A_{7}\right] \\
& \quad-\mathrm{P}\left[A_{1} A_{2} A_{5} A_{6} A_{7}\right]-\mathrm{P}\left[A_{2} A_{3} A_{5} A_{6} A_{7}\right] \\
& \quad+\mathrm{P}\left[A_{1} A_{2} A_{3} A_{5} A_{6} A_{7}\right]+\mathrm{P}\left[A_{1} A_{2} A_{4} A_{5} A_{6} A_{7}\right] \\
& \quad+\mathrm{P}\left[A_{2} A_{3} A_{4} A_{5} A_{6} A_{7}\right]-\mathrm{P}\left[A_{1} A_{2} A_{3} A_{4} A_{5} A_{6} A_{7}\right]
\end{aligned}
$$

For independent interference we have

$$
\begin{aligned}
\mathrm{P}\left[B_{\mathrm{MHA}}\right]=\left(1-\mathrm{P}\left[A_{2}\right]\right) \\
\quad\left(\mathrm{P}\left[A_{1}\right]\left(\mathrm{P}\left[A_{4}\right]+\mathrm{P}\left[A_{5}\right] \mathrm{P}\left[A_{6}\right]-\mathrm{P}\left[A_{4}\right] \mathrm{P}\left[A_{5}\right] \mathrm{P}\left[A_{6}\right]\right)\right) \\
+\mathrm{P}\left[A_{2}\right]\left(\mathrm{P}\left[A_{7}\right]+\left(1-\mathrm{P}\left[A_{7}\right]\right)\right. \\
\quad\left(\mathrm{P}\left[A_{1}\right]+\mathrm{P}\left[A_{3}\right]-\mathrm{P}\left[A_{1}\right] \mathrm{P}\left[A_{3}\right]\right) \\
\left.\quad\left(\mathrm{P}\left[A_{4}\right]+\mathrm{P}\left[A_{5}\right] \mathrm{P}\left[A_{6}\right]-\mathrm{P}\left[A_{4}\right] \mathrm{P}\left[A_{5}\right] \mathrm{P}\left[A_{6}\right]\right)\right)
\end{aligned}
$$

The probabilities in the last equations can be again calculated by applying Equation (8).

Figure 9 compares the packet delivery probabilities of multi-hop-aware cooperative relaying and conventional cooperative relaying. The position of $S$ is at the origin, while $D_{1}$ and $D_{2}$ have $x$-coordinates of 1 and 2 , respectively. The location of the relay used in the first hop is altered while the position of the second relay is kept fixed at 1.5. As we can see, the optimal position of the first relay is at about 0.5 , i.e., in the middle between $S$ and $D_{1}$. Furthermore, the 
plot shows that the gain for the additional link (Link 7 in Figure 2) is very small for the considered scenario.

Next we want to see if the gain of MHA relaying improves when we consider independent interference. Figure 10 compares the performance of multi-hop-aware cooperative relaying and conventional two-hop relaying for the dependent and independent interference cases. The upper two curves show the performance of the two relaying schemes for independent interference. Here, the performance of MHA cooperative relaying is significantly better than the performance of two-hop relaying. Again, if we consider dependent interference, the performance improvement is much smaller. These results show that the performance gain of MHA cooperative relaying is significantly reduced if we consider a model of dependent interference.

\section{RELATED WORK}

\subsection{Temporal and Spatial Properties of Inter- ference}

A number of recent papers aim to analyze the temporal and spatial characteristics of interference. In particular, Haenggi introduced the 'uncertainty cube', which models the stochastic aspects of the network under consideration [7]. The author also derives the correlation of interference for network scenarios represented by the vertices of this cube.

The authors of [17] extended the results of Haenggi by considering a wider set of sources of correlation, including the nodes' locations, the temporal correlation of the wireless channel and the particular traffic pattern adopted by communicating stations.

This work is also strongly related to the analysis in [3], where Ganti et al. derive the conditional probability of outage situations at different time slots for scenarios that exhibit correlated interference. In particular, our work adapts and extends some aspects of the above-cited work to the spatial domain by addressing cooperative communications.

\subsection{Performance of Cooperative Communica- tion Systems}

The performance of cooperative techniques has been extensively investigated in recent years, and some of the contributions that are closer to the analysis contained in this work are briefly reviewed in the following.

Gupta and Kumar derive the capacity of the relay-aided wireless channel assuming point-to-point coding [6]. Their analysis is extended by Gastpar and Vetterli in [4], which accounts for more general channel access schemes and coding solutions, where the single relays are allowed to help each other in a 'network coding' fashion.

Laneman et al. address the design of cooperative protocols including outage probability as a performance metric [15]. The authors show that the proposed relaying schemes are capable of performing within $1.5 \mathrm{~dB}$ from the optimum and provide a significant resource gain compared to non-cooperative solutions.

Amplify-and-Forward schemes, where the relay does not decode the signal received from the transmitting station, are analyzed in [12], where Nakagami- $m$ fading is assumed. The authors derive the outage probability characterizing the considered network scenario, and verify that the analysis closely matches the simulated performance.
The outage probability of coded cooperation schemes, where relays follow an incremental forwarding rule, instead of simply retransmitting the same information sent by the transmitter, is studied in [11]. The authors show that coded cooperation is fundamentally different from decodeand-forward schemes, and they further prove that such a forwarding technique is beneficial for the network performance compared to non-cooperative and repetitive solutions.

Incremental systems are also studied in [2], where the authors obtain the throughput, delay and outage performance of cooperative coded systems consisting of multiple transmissions. The analysis of [2] is not based on capacity considerations, but it relies on the sphere-packing bound and hence accounts for the effect of the number of symbols that are transmitted in each attempt, without loosing generality since the bound is not limited to using a particular encoding and decoding engine.

All the above-mentioned works characterize in detail the performance of the cooperative link, though they do not consider the influence of interference from external stations, which is the ultimate aim of this contribution. Hence, the presented paper may be seen as an effort for extending previous theoretic works to the interference limited scenario.

\subsection{Influence of Interference on Cooperative Communications}

Despite extensive research efforts on cooperative communications, few papers considered the influence of interference on the performance of a cooperative scenario. The most recent contributions are summarized in the following.

A look at cooperation between the base stations of different cells grouping users is presented in [5], though that work is quite distant from the single link point of view analyzed in this contribution. Particularly, the authors investigate the possibility of enabling cooperative decoding of signals received at different base stations, for the sake of obtaining an increased performance by exploiting interference in order to create a virtual multiple-input multiple-output channel.

A capacity bound for scenarios consisting of two transmitter-receiver pairs is studied in [16], assuming that stations from the different pairs may cooperate, though they cause interference to the other communicating pairs. The paper shows that a significant performance increase is achieved when the two transmitters are allowed to cooperate following the rule proposed by the authors, and further proves that the capacity bound is achieved when the cooperation channel gain is infinite.

Reference [20] studies the sum-rate and energy efficiency of a cooperative network and highlights a tradeoff between the throughput gain provided by cooperative communications and the amount of interference introduced by relay stations in an asynchronous multi-user scenario. The authors derive through numerical analysis and simulations the optimal size of a cooperative region for maximizing the network sum-rate and energy efficiency.

The performance of decode-and-forward relaying schemes is analyzed in [13] assuming that the cooperative link is affected by co-channel interference. Particularly, the authors derive the outage probability of the system and provide optimal energy allocation strategies under an adaptive relay selection assumption, where only stations that successfully decoded the transmission of the source node may cooperate.

The works summarized above are a step toward analyzing 
the behavior of cooperation when the communication is affected by interfering nodes, though the results obtained do not consider the correlation properties of interference, which may significantly influence the network performance. This paper aims at extending the above-cited references by introducing spatial correlation of interference experienced by nodes at different locations, hence providing a more accurate analysis of cooperative techniques in interference limited networks.

\section{CONCLUSIONS AND FURTHER WORK}

In the paper at hand we derived the joint probabilities of successful packet delivery at multiple receivers under spatially and temporally dependent interference. This tool was then applied to selected cooperative relaying scenarios. We showed how the performance of cooperative relaying changes under dependent interference compared to the case of independent interference. For good communication conditions, dependence decreases the end-to-end performance, while for harsh communication conditions it increases the performance. We analyzed the probability of full packet delivery at a given slot for repeated cooperative relaying. The outcome was as follows: repetitions of the relaying process significantly improve packet delivery for independent interference; this gain can be smaller for dependent interference.

Next, a scheme called multi-hop-aware cooperative relaying was analyzed. We showed that the performance gain of this protocol is reduced when interference exhibits high temporal and spatial dependencies. Overall, results show that the spatial and temporal dependence exhibited by interference has a large impact on the performance of cooperative relaying. Many related works, however, derive performance metrics without considering the dependence of interference.

As a next step, we plan to generalize our work to the case of multiple relays. Here, some relay selection protocol can be assumed to choose the best relay with regard to some metric. Additionally, we will analyze the performance of maximum ratio combining using the same tools.

\section{Acknowledgments}

This work has been supported by the Austrian Science Fund (FWF) under grant P24480-N15 and by the ERDF, $\mathrm{KWF}$, and the state of Austria under grants KWF20214/15935/23108 (RELAY) and 20214/20777/31602 (Research Days). It has been performed in the research cluster Lakeside Labs. The work of S. Toumpis has been partly done during a research stay at Lakeside Labs GmbH.

\section{REFERENCES}

[1] H. Adam, C. Bettstetter, and S. M. Senouci. Multi-hop-aware cooperative relaying. In Proc. IEEE Vehicular Technology Conf. (VTC), Barcelona, Spain, Apr. 2009.

[2] F. Babich and A. Crismani. Cooperative coding schemes: Design and performance evaluation. IEEE Trans. Wireless Commun., 11(1):222-235, Jan. 2012.

[3] R. Ganti and M. Haenggi. Spatial and temporal correlation of the interference in ALOHA ad hoc networks. IEEE Commun. Lett., 13(9):631-633, Sept. 2009.

[4] M. Gastpar and M. Vetterli. On the capacity of wireless networks: the relay case. In Proc. IEEE
Intern. Conf. on Communications (ICC), pages 1577-1586, June 2002.

[5] D. Gesbert, S. Hanly, H. Huang, S. Shamai Shitz, O. Simeone, and W. Yu. Multi-cell MIMO cooperative networks: a new look at interference. IEEE J. Select. Areas Commun., 28(9):1380-1408, Dec. 2010.

[6] P. Gupta and P. R. Kumar. The capacity of wireless networks. IEEE Trans. Inform. Theory, 46:388-404, 2000.

[7] M. Haenggi. Outage, local throughput, and capacity of random wireless networks. IEEE Trans. Commun., 8(8), Aug. 2009.

[8] M. Haenggi. Stochastic Geometry for Wireless Networks. Cambridge University Press, 2013.

[9] M. Haenggi and R. Ganti. Interference in Large Wireless Networks. now publishing, 2009.

[10] R. Hekmat and P. Van Mieghem. Interference in wireless multi-hop ad-hoc networks and its effect on network capacity. Wirel. Netw., 10(4):389-399, July 2004.

[11] T. Hunter, S. Sanayei, and A. Nosratinia. Outage analysis of coded cooperation. IEEE Trans. Inform. Theory, 52(2):375-391, Feb. 2006.

[12] S. S. Ikki and M. Ahmed. Performance analysis of cooperative diversity wireless networks over Nakagami-m fading channel. IEEE Commun. Lett., 11(4):334-336, Apr. 2007.

[13] S. S. Ikki, P. Ubaidulla, and S. Aissa. Regenerative cooperative diversity networks with co-channel interference: performance analysis and optimal energy allocation. IEEE Trans. Veh. Technol., 62(2):896-902, Feb. 2013.

[14] A. Iyer, C. Rosenberg, and A. Karnik. What is the right model for wireless channel interference? IEEE Trans. Wireless Commun., 8(5):2662-2671, 2009.

[15] J. N. Laneman, D. N. C. Tse, and G. W. Wornell. Cooperative diversity in wireless networks: efficient protocols and outage behavior. IEEE Trans. Inform. Theory, 50:3062-3080, 2004.

[16] Y. Peng and D. Rajan. Capacity bounds of half-duplex Gaussian cooperative interference channel. In Proc. IEEE Intern. Symp. on Inform. Theory (ISIT), pages 2081-2085, July 2009.

[17] U. Schilcher, C. Bettstetter, and G. Brandner. Temporal correlation of interference in wireless networks with Rayleigh fading. IEEE Trans. on Mobile Computing, 11(12):2109-2120, Dec. 2012.

[18] A. Sendonaris, E. Erkip, and B. Aazhang. User cooperation diversity - Part I: system description. IEEE Trans. Commun., 51:1927-1938, Nov. 2003.

[19] D. Stoyan, W. S. Kendall, and J. Mecke. Stochastic Geometry and Its Applications, chapter 2.4. John Wiley \& Sons Ltd, 1995.

[20] S. Vakil and B. Liang. Cooperative diversity in interference limited wireless networks. IEEE Trans. Wireless Commun., 7(8):3185-3195, Aug. 2008.

[21] Y. Zhong, W. Zhang, and M. Haenggi. Managing interference correlation through random medium access. 2013. Submitted. Available at http://www.nd.edu/ ${ }^{\sim m h a e n g g i / p u b s / t w c 13 c . p d f, ~}$ accessed on May 7th, 2013. 